\title{
Optimization of sensors based on multimode interference in single- mode-multimode-single-mode structure
}

\author{
Ignacio Del Villar, Abian B. Socorro, Jesus M. Corres, Francisco J. Arregui, Member, IEEE, and Ignacio R. Matias, \\ Senior Member, IEEE
}

\begin{abstract}
By coating a single-mode-multimode-single-mode (SMS) structure with a complex refractive index thin-film the central wavelength of the transmission and attenuation bands of the optical spectrum can be shifted. By adequate selection of multimode segment length and diameter, the thin-film thickness and the thin-film refractive index, the sensitivity of the device to the surrounding medium refractive index, the thickness of the thin-film or the refractive index of the thin-film can be optimized. Experimental results are corroborated numerically with the Transfer-Matrix-Method.
\end{abstract}

Index Terms - optical fiber interference, thin films, optical fiber transducers, SMS structure

\section{INTRODUCTION}

$\mathrm{S}$ ingle-mode - multimode - single-mode (SMS) fiber structure is a well-known optical configuration, which is obtained by splicing a multimode fiber segment (coreless MMF) between two single-mode fiber (SMF) segments.

The basic physical principle of the device is that light transmitted through the fundamental mode of the SMF segment is coupled to several modes into the MMF section and recoupled to the fundamental mode of the SMF at the end of the MMF segment [1]. Due to the phenomenon of multimodal interferometry (MMI) [2], both transmission and attenuation bands are obtained in the optical spectrum $[1,3]$.

Multimode interferometry in SMS devices can be used for the development of interesting applications [4-5]. In the research domain of sensors, devices have been developed that permit to detect displacement, strain, pressure, temperature or refractive index [6-10].

The adsorption of thin-films on other optical structures such as cladding removed multimode optical fiber [11], long-period fiber gratings [12], tapered optical fiber [13], hollow core fiber [14] or photonic crystal fiber [15] expand the traditional domains where optical fiber is used. Some examples of this are the detection of ambient compounds (gases, moisture) [1617], development of chemical, biological and medical applications [18-19]. Consequently, SMS structure should also be a good platform for the fabrication of simple and costeffective devices, which can be used for the above mentioned applications.

However, very little attention has been paid to thin-film coated SMS structures. A theoretical study indicates the possibility to increase the sensitivity to refractive index by coating the multimode segment [3]. In addition to this, another publication has shown both theoretically and experimentally

Manuscript received June 14, 2013. This work was supported in part by the Spanish Ministry of Education and Science-FEDER TEC2006-12170 and TEC2007-67987-C02-02/MIC.

The authors are with the Electrical and Electronic Engineering Department, Public University of Navarra, 31006 Pamplona, Spain (e-mail: ignacio.delvillar@unavarra.es). that it is possible to obtain a wavelength shift of the transmission and attenuation bands if a thin-film is progressively deposited on the coreless MMF segment, and that the sensitivity to thin-film thickness variations increases if the mode transition region is approached, like in long-period fiber gratings [20].

In this work, a thorough analysis in a transmission configuration is presented. The influence of parameters such as MMF segment length and diameter, thin-film refractive index and thickness and surrounding medium refractive index towards the development of optimized devices are studied. The extension of the design rules to a reflective configuration [21], can be done by simply considering SMS structures with middle length of the MMF segment and a mirror at one extreme of the segment, something that has already been proved experimentally for LPFGs [22].

In section 2 the simulation method for the analysis of SMS structures is explained. In section 3 the procedure used for the experimental results is detailed. Numerical and experimental data are presented and discussed in section 4. Finally, some conclusions are included in section 5.

\section{THEORY}

In order to obtain the optical transmission through the SMS structure, a simulation tool is developed based on the transfer matrix method [23]. The procedure for obtaining the transmission as a function of wavelength is based on three steps:

\section{A. Calculation of the propagation constants}

The application of the transfer matrix method presented in [24], permits to calculate the propagation constants of modes in a cylindrical waveguide by expressing their fields in terms of radial and azimuthal vector components. In the SMS structure of Fig. 1 there are two different waveguides, whose propagation constants are calculated. In view of the azimuthal symmetry of the waveguides, only modes with azimuthal $\operatorname{order} v=1$ are considered for the analysis [25]. Consequently, the notation for modes will be of the type ' $1, \mathrm{j}$ ' (the first subindex representing the azimuthal order, and the second subindex indicating the $\mathrm{j}^{\text {th }}$ mode).

\section{B. Derivation of the transmission and reflection coefficients}

Considering in Fig. 1 the SMF on the left side of the SMS structure as waveguide ' $a$ ', the coreless MMF as waveguide ' $\mathrm{b}$ ', and the SMF on the right side as waveguide ' $\mathrm{c}$ ', the transmission and reflection coefficients can be obtained with this general equation: 


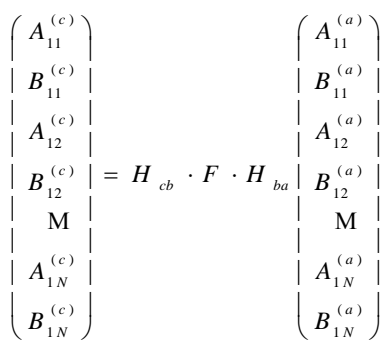

where $A_{1 j}(a)$ is the normalized forward amplitude of the $j$ mode in waveguide ' $a$ ', $A_{1 j}(c)$ is the normalized forward amplitude of the $\mathrm{j}$ mode in waveguide ' $c$ ', $\mathrm{B}_{1 \mathrm{j}}(\mathrm{a})$ is the normalized backward amplitude of the $\mathrm{j}$ mode in waveguide ' $a$ ', and $B_{1 j}(c)$ is the normalized backward amplitude of the $\mathrm{j}$ mode in waveguide 'c'.

$\mathrm{H}_{\mathrm{ba}}$ and $\mathrm{H}_{\mathrm{cb}}$ are the matrices that relate the amplitudes of the modes at both sides of the interface between waveguide ' $b$ ' and ' $a$ ', and between waveguide ' $c$ ' and ' $b$ ' respectively. As an example of both expressions, according to [23], matrix $\mathrm{H}_{\mathrm{cb}}$, which relates the amplitude of the modes at both sides of the interface between waveguide $\mathrm{c}$ and $\mathrm{b}$, can be expressed as indicated in expression (2) at the bottom of this page, where $I_{1 j, 1 k}$ and $J_{1 j, 1 k}$ are the following expressions:

$$
\begin{aligned}
& I_{1 k, 1 j}=\sqrt{P_{1 j}^{-1} P_{1 k}^{-1}} \frac{1}{2} \operatorname{Re} \int_{\phi=0}^{2 \pi} d \phi \int_{r=0}^{\infty}\left[E_{1, j-r}^{b}(r) H_{1, k-\phi}^{a}(r)^{*}-H_{1, k-r}^{a}(r)^{*} E_{1, j-\phi}^{b}(r)\right] d r \\
& J_{1 k, 1 j}=\sqrt{P_{1 j}^{-1} P_{1 k}^{-1}} \frac{1}{2} \operatorname{Re} \int_{\phi=0}^{2 \pi} d \phi \int_{r=0}^{\infty}\left[E_{1, j-r}^{a}(r)^{*} H_{1, k-\phi}^{b}(r)-H_{1, k-r}^{b}(r) E_{1, j-\phi}^{a}(r)^{*}\right] d r
\end{aligned}
$$

In expressions (3) and (4) $\mathrm{E}_{1, \mathrm{j}-\mathrm{r}}$ is the radial electric field of mode $_{1, j}, E_{1, j-\phi}$ is the azimuthal electric field of mode $1, \mathrm{j}, \mathrm{H}_{1, \mathrm{j}-\mathrm{r}}$ is the radial magnetic field of mode $_{1, j}, \mathrm{H}_{1, \mathrm{j}-\phi}$ is the azimuthal magnetic field of $\operatorname{mode}_{1, j}$, and $\mathrm{P}_{1, \mathrm{j}}$ is the normalized power of the same mode.

The expression for matrix $\mathrm{F}$, which is the free propagation matrix over the MMF region of length $\mathrm{L}$ is:
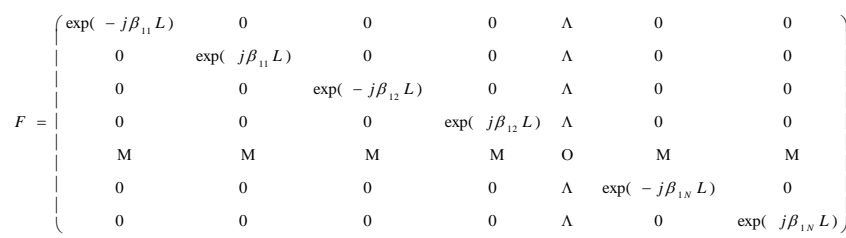

where $\beta_{1 \mathrm{j}}$ is the propagation constant of $\operatorname{mode}_{1, \mathrm{j}}$.

\section{Derivation of the transmission and reflection coefficients}

The transmission can be found by assuming in expression (1) that only one mode is incident $\left(\mathrm{A}^{(\mathrm{a})}{ }_{1,1}=1\right.$ and $\mathrm{A}^{(\mathrm{a})}{ }_{1,2}=\ldots$. $=\mathrm{A}^{(\mathrm{a})}{ }_{1, \mathrm{~N}}=0$ ) and that there is no backward reflection in waveguide ' $c$ ' $\left(\mathrm{B}^{(\mathrm{c})}{ }_{1,1}=\mathrm{B}^{(\mathrm{c})}{ }_{1,2}=\ldots . .=\mathrm{B}^{(\mathrm{c})}{ }_{1, \mathrm{~N}}=0\right)$, and solving the equation. After that, the transmission power can be expressed as:

$$
T=\left|A_{11}^{c}\right|^{2}
$$

\section{EXPERIMENTAL SECTION}

Two SMS structures were fabricated by splicing a segment of coreless multimode fiber (MMF) from POFC Inc. to two standard SMF pigtails from Telnet Inc (see Fig. 1). In one of the structures the diameter of the fibers was $125 \mu \mathrm{m}$, whereas in the other one it was $61.5 \mu \mathrm{m}$.

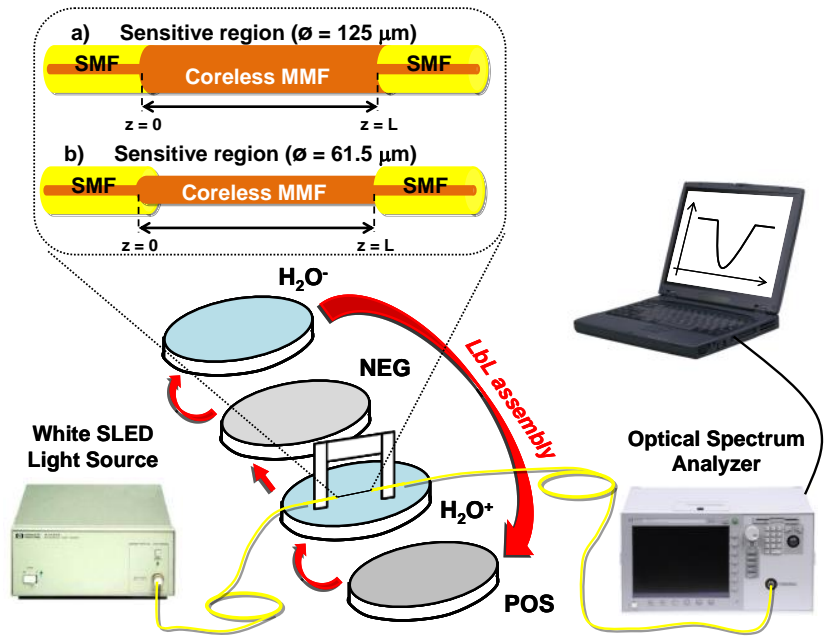

Fig. 1. Experimental setup

For the deposition of the thin-film, layer-by-layer assembly process (LbL) was used, which consists in the deposition of bilayers by electrostatic attraction. In Fig. 1 POS is the positively charged monolayer, NEG is the negatively charged monolayer and $\mathrm{H}_{2} \mathrm{O}^{+}$and $\mathrm{H}_{2} \mathrm{O}^{-}$are the rinsing solutions [26]. The thickness per bilayer was estimated by using the method described in [27].

(5) Two different films were deposited. For the first one, each bilayer of the coating was obtained by immersing the substrate in two solutions of poly (allylamine hydrochloride) (PAH) and poly (acrylic acid) (PAA), both of them in $10 \mathrm{mM}$ concentration and adjusted to $\mathrm{pH}$ 4.5. These polymers were

$$
\begin{aligned}
& \text { Equation (2) } \\
& \left|\begin{array}{c}
A_{11}^{(c)} \\
B_{11}^{(c)} \\
A_{12}^{(c)} \\
B_{12}^{(c)} \\
\mathrm{M} \\
A_{0 N}^{(c)} \\
B_{1 N}^{(c)}
\end{array}\right|= \\
& \left\{\begin{array}{c}
\frac{1}{2}\left(I_{11,11}+J_{11,11}\right) \\
\frac{1}{2}\left(I_{11,11}-J_{11,11}\right) \\
\frac{1}{2}\left(I_{12,11}+J_{12,11}\right) \\
\frac{1}{2}\left(I_{12,11}-J_{12,11}\right) \\
\mathrm{M} \\
\frac{1}{2}\left(I_{1 N, 11}+J_{1 N, 11}\right) \\
\frac{1}{2}\left(I_{1 N, 11}-J_{1 N, 11}\right)
\end{array}\right. \\
& \frac{1}{2}\left(I_{11,11}-J_{11,11}\right) \\
& \frac{1}{2}\left(I_{11,12}+J_{11,12}\right) \\
& \frac{1}{2}\left(I_{11,11}+J_{11,11}\right) \\
& \frac{1}{2}\left(I_{11,12}-J_{11,12}\right) \\
& \frac{1}{2}\left(I_{12,11}-J_{12,11}\right) \\
& \frac{1}{2}\left(I_{12,12}+J_{12,12}\right) \\
& \frac{1}{2}\left(I_{11,12}-J_{11,12}\right) \quad \Lambda \\
& \frac{1}{2}\left(I_{11,12}+J_{11,12}\right) \quad \Lambda \\
& \frac{1}{2}\left(I_{11,1 N}+J_{11,1 N}\right) \\
& \frac{1}{2}\left(I_{11,1 N}+J_{11,1 N}\right) \\
& \frac{1}{2}\left(I_{12,12}-J_{12,12}\right) \quad \Lambda \quad \frac{1}{2}\left(I_{12,1 N}+J_{12,1 N}\right) \\
& \frac{1}{2}\left(I_{12,11}+J_{12,11}\right) \\
& \frac{1}{2}\left(I_{12,12}-J_{12,12}\right) \\
& \frac{1}{2}\left(I_{12,12}+J_{12,12}\right) \quad \Lambda \\
& \mathrm{M} \\
& \mathrm{M} \\
& \frac{1}{2}\left(I_{12,1 N}+J_{12,1 N}\right) \\
& \mathrm{M} \\
& \frac{1}{2}\left(I_{1 N, 11}-J_{1 N, 11}\right) \quad \frac{1}{2}\left(I_{1 N, 12}+J_{1 N, 12}\right) \quad \frac{1}{2}\left(I_{1 N, 12}-J_{1 N, 12}\right) \quad \Lambda \\
& \frac{1}{2}\left(I_{1 N, 1 N}+J_{1 N, 1 N}\right) \\
& \frac{1}{2}\left(I_{1 N, 1 N}+J_{1 N, 1 N}\right) \\
& \begin{array}{c}
\frac{1}{2}\left(I_{11,1 N}-J_{11,1 N}\right) \\
\frac{1}{2}\left(I_{11,1 N}+J_{11,1 N}\right) \\
\frac{1}{2}\left(I_{12,1 N}+J_{12,1 N}\right) \\
\frac{1}{2}\left(I_{12,1 N}+J_{12,1 N}\right) \\
\mathrm{M} \\
\frac{1}{2}\left(I_{1 N, 1 N}+J_{1 N, 1 N}^{(b)}\right) \\
\frac{1}{2}\left(I_{1 N, 1 N}+J_{1 N, 1 N}^{(b)}\right)
\end{array}
\end{aligned}
$$


purchased from Sigma-Aldrich. For the second nanocoating, a PSS water solution was used as anionic polyelectrolyte and a solution of Titanium(IV) oxide nanoparticles in ultrapure water was used as cationic solution. The $\mathrm{pH}$ of the rinsing solutions and both anionic and cationic solutions was adjusted to 2.0 [28].

As it is plotted in Fig. 1, a super luminescent emitting diode light source (HP_83437A - SLED from Agilent Inc.) launches light in a wavelength range between 1150 and $1680 \mathrm{~nm}$ into the SMS structure. At the other side, the light modulated by the SMS is detected by a HP-86142A optical spectrum analyzer (OSA) from Agilent Inc.

\section{RESULTS}

Propagation of light in an SMS structure is based on coupling of light from the input SMF to several modes in the MMF segment. Light propagates trough different modes in the MMF segment and, hence, the phase of the modes is different at the point where light is coupled to the output SMF segment.

In addition to this, there are several planes where constructive interference occurs, which may be caused by a fractional constructive interference or by a light field condensation between the several guided modes in a particular plane (the self-imaging effect) [10]. The last option permits to obtain a higher transmission than in the rest of cases. Consequently, the selection of the MMF section length is focused on monitoring the evolution of the band produced by the self-imaging effect.

To this purpose, the application of the following analytical expression permits to obtain the MMF section length adequate for setting the first self-image band at a specific wavelength [10]:

$$
Z_{i}=\frac{4 D^{2} n}{\lambda}
$$

where $D$ and $n$ are the MMF diameter and refractive index, respectively, and $\lambda$ is the operational wavelength.

In view of the wavelength range that is monitored with the experimental setup of Fig. 1, a good option is to fix the transmission band at the third optical communications window: $1550 \mathrm{~nm}$. At this wavelength the refractive index of silica is 1.444 , which can be applied to variable $n$. So the length of the MMF segment should be $58 \mathrm{~mm}$.

In Fig. 2 the numerical method presented in section 2 is used to analyze the evolution of the optical spectrum when a nanocoating of PAH/PAA (see section 2) is deposited on the MMF section. When the nanocoating thickness increases the transmission and attenuation bands present a wavelength shift which rate is maximum in the proximity of a fading region (450 to $750 \mathrm{~nm}$ ). This fading region is caused by coupling to a lossy guided mode in the nanocoating [11]. After the fading region the bands reappear and experiment a wavelength shift rate that is reduced as the coating increases.

In Fig. 3 the experimental results of a SMS structure with a MMF segment of $58 \mathrm{~mm}$ are shown and they agree well with those of Fig. 2. In order to avoid a mismatching between the experimental and the theoretical results it is very important to control precisely the length of the MMF.

The length of the MMF section $(58 \mathrm{~mm})$ is not practical in terms of miniaturization of the final sensing device. Consequently, in order to reduce the length of the device, another SMS structure was fabricated with an MMF segment length of $20 \mathrm{~mm}$, which is not a value that permits to obtain in the spectral range analyzed a transmission band caused but a self-image. In fact the SMS structure with a $58 \mathrm{~mm}$ MMF section permits to obtain in the optical spectrum a transmission band caused by the first-self image and all transmission bands obtained for lower values are caused by fractional constructive interference. However, according to [20], the theoretical and experimental results of Fig. 4 and 5 show that the obtained transmission bands experiment a similar wavelength shift to that observed in Fig. 2 and 3.

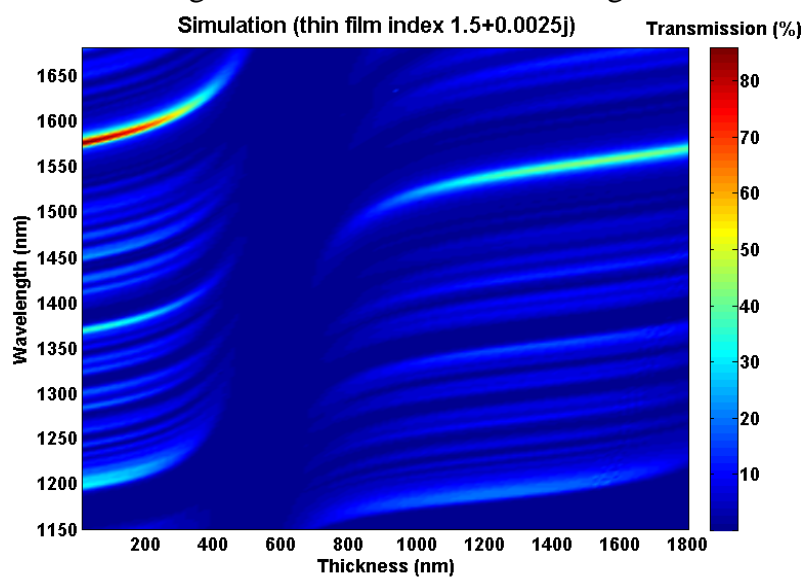

Fig. 2. Evolution of the optical spectrum for an SMS structure deposited with a PAH/PAA nanocoating. MMF segment diameter $125 \mu \mathrm{m}$ and length $58 \mathrm{~mm}$. Numerical results obtained as a function of nanocoating thickness (thin-film refractive index $1.5+0.0025 j$ ).

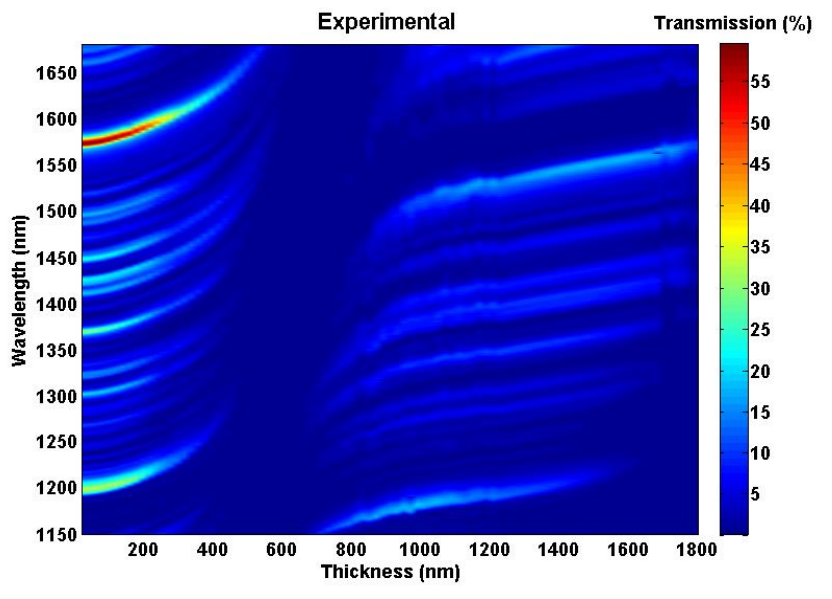

Fig. 3. Evolution of the optical spectrum for an SMS structure deposited with a PAH/PAA nanocoating. MMF segment diameter $125 \mu \mathrm{m}$ and length $58 \mathrm{~mm}$. Experimental results (bilayer thickness: $19.8 \mathrm{~nm}$ ).

In order to compare the results of both $20 \mathrm{~mm}$ and $58 \mathrm{~mm}$ length devices, in Fig. 6 the central wavelength of the transmission bands is represented both theoretically and experimentally (the gray plots correspond to the $20 \mathrm{~mm}$ SMS structure whereas the black plots correspond to the $58 \mathrm{~mm}$ 
SMS structure). It is easy to observe that the wavelength shift rate is similar independent of the length of the structure.

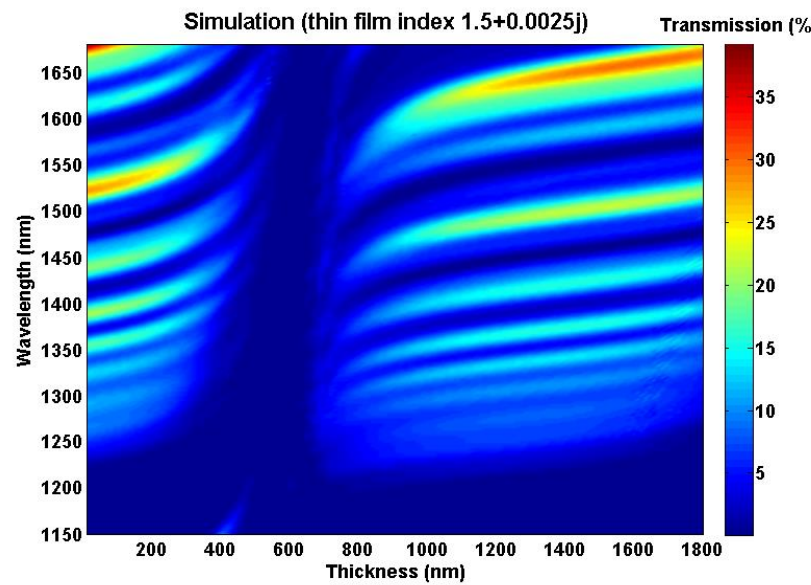

Fig. 4. Evolution of the optical spectrum for an SMS structure deposited with a PAH/PAA nanocoating. MMF segment diameter $125 \mu \mathrm{m}$ and length $20 \mathrm{~mm}$. Numerical results obtained as a function of nanocoating thickness (thin-film refractive index $1.5+0.0025 j$ ).

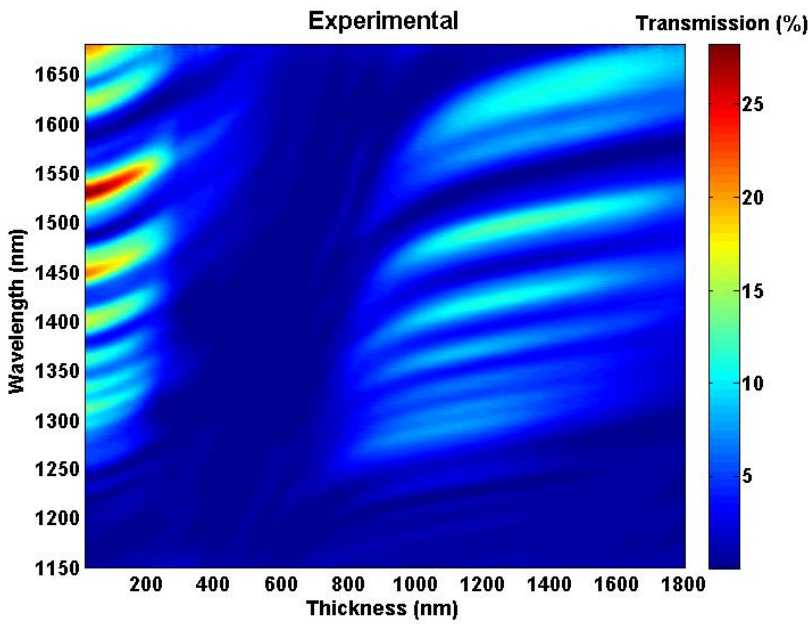

Fig. 5. Evolution of the optical spectrum for an SMS structure deposited with a PAH/PAA nanocoating. MMF segment diameter $125 \mu \mathrm{m}$ and length $20 \mathrm{~mm}$. Experimental results (bilayer thickness: $15 \mathrm{~nm}$ ).

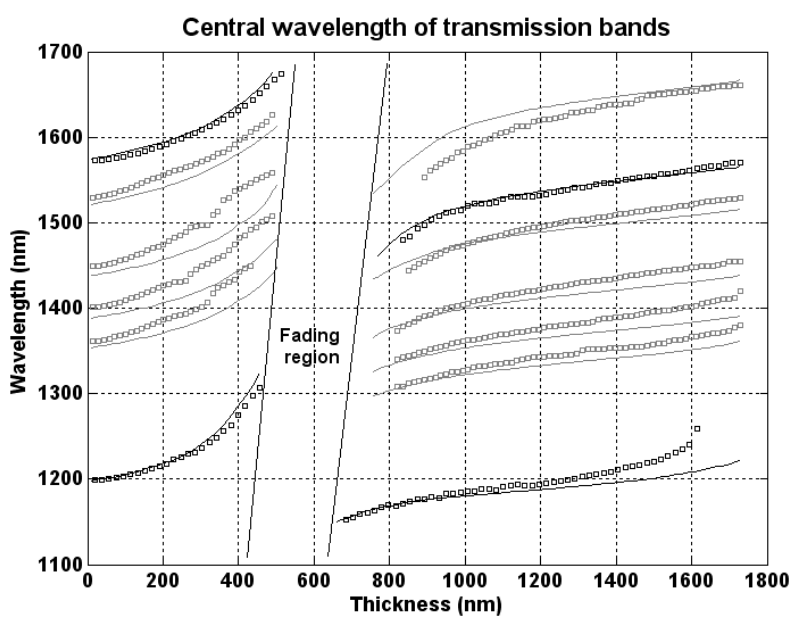

Fig. 6. Evolution as a function of thickness of the central wavelength of transmission bands obtained with an SMS structure of $58 \mathrm{~mm}$ (black plots) and with an SMS structure of $20 \mathrm{~mm}$ (grey plots). Continuous line (simulation data); squares (experimental data).
Once it has been observed that wavelength shift rate is independent of the length of the device, focus will be centered now on the refractive index of the material. PAH/PAA is a material with a refractive index 1.5. However, higher refractive index materials can be deposited such as $\mathrm{TiO}_{2} / \mathrm{PSS}$.

In Fig. 7 and 8 the effect of a $\mathrm{TiO}_{2} / \mathrm{PSS}$ thin-film deposited on a $20 \mathrm{~mm}$ length SMS structure is presented both theoretically and experimentally.

The three regions already observed in Figs. 2-5 can be distinguished. However, the wavelength shift ratio is higher. This fact is confirmed by comparing in Fig. 9a and Fig. 9b the central wavelength of the transmission bands of two SMS structures: the first one with an MMF section of $20 \mathrm{~mm}$ deposited with PAH/PAA and second one with an MMF section of $20 \mathrm{~mm}$ deposited with $\mathrm{TiO}_{2} / \mathrm{PSS}$. The selection of a material with a refractive index that approximates that of the substrate increases the wavelength shift. As an example, the self-image band moves from $1521 \mathrm{~nm}$ to $1613.7 \mathrm{~nm}$ with PAH/PAA and from $1521 \mathrm{~nm}$ to $1568.6 \mathrm{~nm}$ with $\mathrm{TiO}_{2} / \mathrm{PSS}$. However, the scale of the $\mathrm{x}$ axis is different for PAH/PAA and $\mathrm{TiO}_{2} / \mathrm{PSS}$. If we calculate sensitivity to variations in the nanocoating thickness as the wavelength shift divided by nanocoating thickness range, this parameter is lower with PAH/PAA $(92.7 \mathrm{~nm} / 504 \mathrm{~nm}=0.184)$ than with $\mathrm{TiO}_{2} / \mathrm{PSS}$ $(47.6 \mathrm{~nm} / 132 \mathrm{~nm}=0.361)$. However, the wavelength shift is non-linear. Consequently, a better knowledge of the sensitivity can be obtained with the derivative of the wavelength as a function of thickness. The maximum derivative is obtained in the proximity of the fading region and it is 0.383 and 0.690 respectively for both $\mathrm{PAH} / \mathrm{PAA}$ and $\mathrm{TiO}_{2} / \mathrm{PSS}$ nanocoatings.

The reason why the deposition of a nanocoating with a higher refractive index leads to a higher sensitivity can be explained by the phenomenon of modal transition [20,29], which basically consists in the guidance of a mode in the nanocoating, which induces a reorganization of the effective indices of the modes in the MMF section. With a higher refractive index in the nanocoating the transition region is achieved faster and it is more abrupt, which causes and increase in the wavelength shift induced by variation in the nanocoating refractive index.

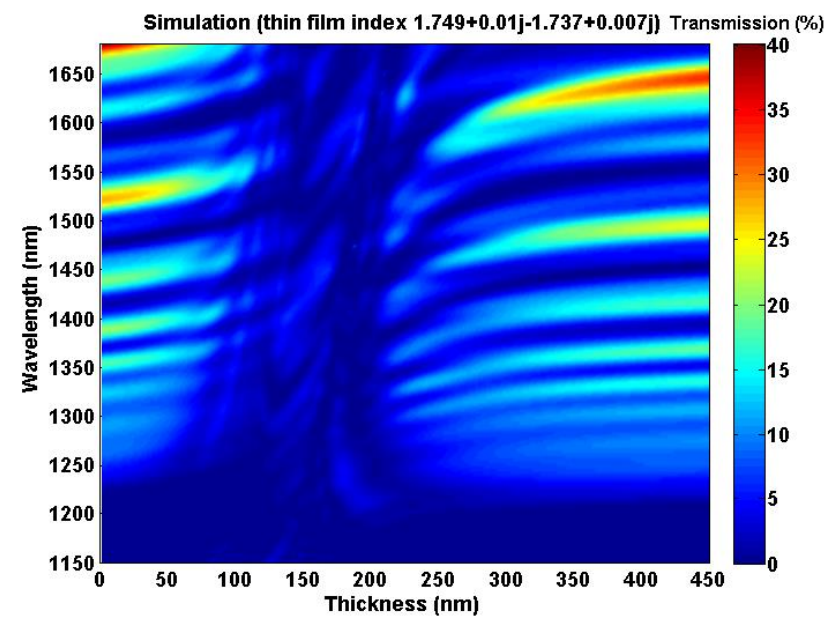

Fig. 7. Evolution of the optical spectrum for an SMS structure deposited with a TiO2/PSS nanocoating. MMF segment diameter $125 \mu \mathrm{m}$ and length $20 \mathrm{~mm}$. Numerical results obtained as a function of nanocoating thickness (thin-film refractive index ranging from $1.749+0.01 \mathrm{j}$ to $1.737+0.007 \mathrm{j}$ in the optical spectrum according to [26]). 


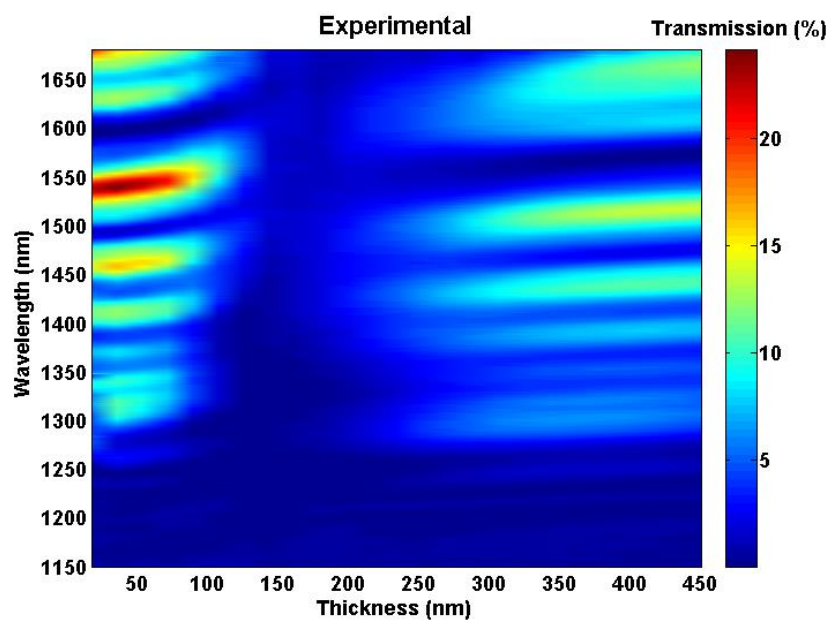

Fig. 8. Evolution of the optical spectrum for an SMS structure deposited with a $\mathrm{TiO}_{2} / \mathrm{PSS}$. nanocoating. MMF segment diameter $125 \mu \mathrm{m}$ and length $20 \mathrm{~mm}$. Experimental results (bilayer thickness: $18 \mathrm{~nm}$ ).
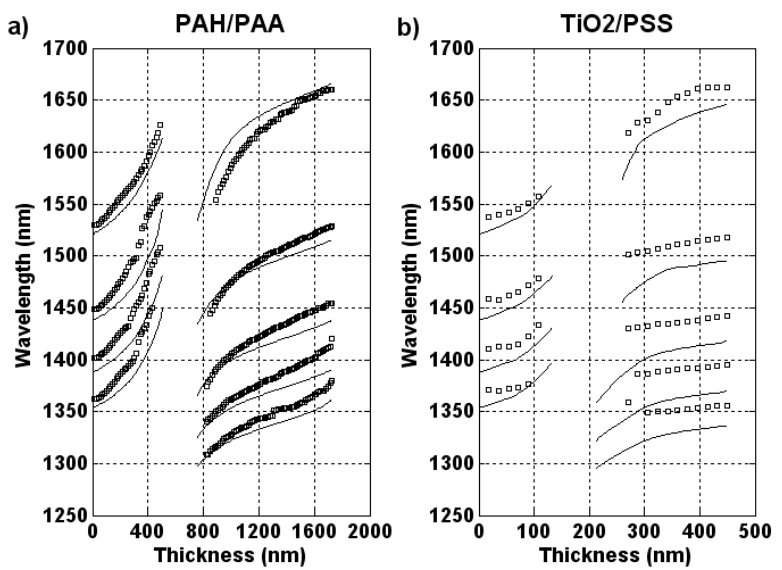

Fig. 9. Evolution as a function of thickness of the central wavelength of transmission bands obtained with an SMS structure of $20 \mathrm{~mm}$. Simulation data, continuous line; experimental data, squares. a) Deposition of PAH/PAA; b) Deposition of $\mathrm{TiO}_{2} / \mathrm{PSS}$.

Once it has been observed that the deposition a material with a higher refractive index than that of the substrate is a better option in terms of sensitivity, another parameter will be analyzed: the diameter of the MMF segment.

In other works it has been observed that reducing the diameter of the MMF segment in an uncoated SMS structure leads to higher sensitivity to refractive index $[1,10]$.

Following this idea, an SMS structure with MMF segment diameter $61.5 \mu \mathrm{m}$ was deposited and coated with PAH/PAA. The length of the device was $15 \mathrm{~mm}$, which permits to visualize the self-image band in the optical spectrum.

As expected, theoretical and experimental results in Fig. 10 and 11 prove that the total wavelength shift is increased by a factor that approximates the ratio between the diameters of the optical fibers analyzed: $125 \mu \mathrm{m} / 61.5 \mu \mathrm{m}$.

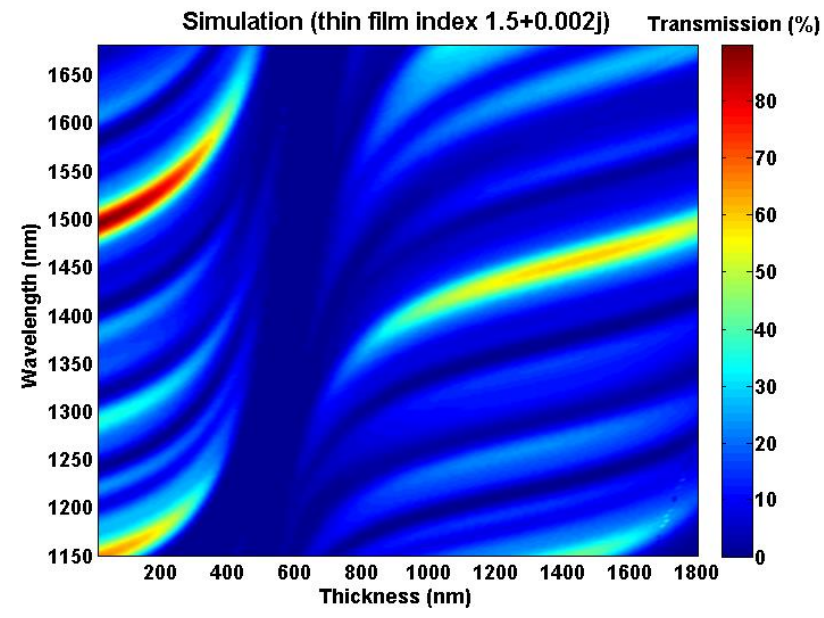

Fig. 10. Evolution of the optical spectrum for an SMS structure deposited with a PAH/PAA nanocoating. MMF segment diameter $125 \mu \mathrm{m}$ and length $58 \mathrm{~mm}$. Numerical results obtained as a function of nanocoating thickness (thin-film refractive index $1.5+0.0025 \mathrm{j}$ ).

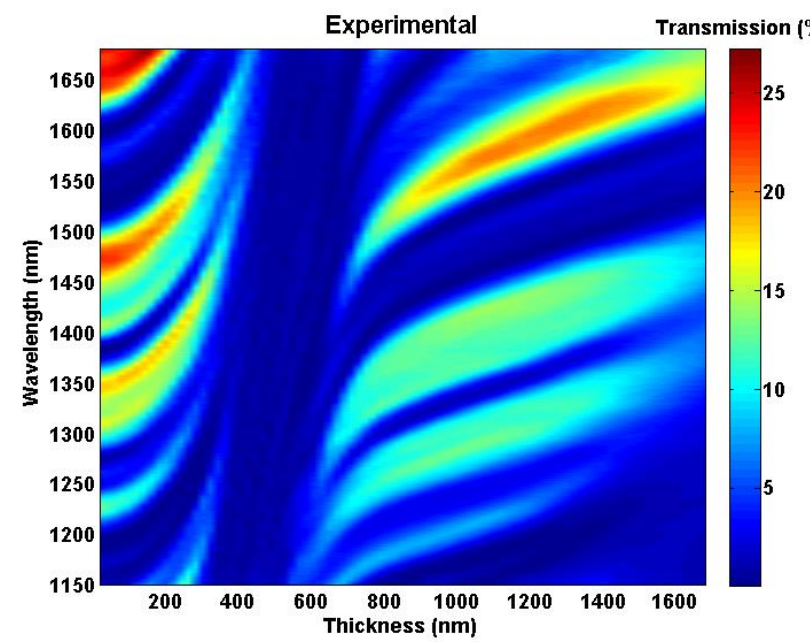

Fig. 11. Evolution of the optical spectrum for an SMS structure deposited with a PAH/PAA nanocoating. MMF segment diameter $125 \mu \mathrm{m}$ and length $58 \mathrm{~mm}$. Experimental results (bilayer thickness: $20 \mathrm{~nm}$ ).

This fact can be better observed by comparing in Fig. 12a and Fig. 12b the central wavelength of the transmission bands of the $58 \mathrm{~mm}$ length SMS with diameter $125 \mu \mathrm{m}$ and the 15 $\mathrm{mm}$ length SMS with diameter $61.5 \mu \mathrm{m}$ as a function of the nanocoating thickness. For example, in Fig. 12a the band located at $1574 \mathrm{~nm}$ for a nanocoating thickness of $0 \mathrm{~nm}$ experiments a wavelength shift of $89.6 \mathrm{~nm}$ if a nanocoating of $468 \mathrm{~nm}$ is deposited, whereas in Fig. 12b the band located at $1494.5 \mathrm{~nm}$ for a nanocoating of thickness $0 \mathrm{~nm}$ experiments a wavelength shift of $172.3 \mathrm{~nm}$. This behavior can be explained by considering the results obtained in [20,29], where it is proved that the resonance wavelength shift is caused by changes in the difference between the effective indices of modes $\mathrm{HE}_{1,1}$ and $\mathrm{HE}_{1,3}$ in the $\mathrm{MMF}$ section. Since the separation between the effective indices of $\mathrm{HE}_{1,1}$ and $\mathrm{HE}_{1,3}$ modes in the MMF section of an uncoated SMS structure with a lower diameter is increased, the wavelength shift is higher as the nanocoating is progressively deposited on the structure. 

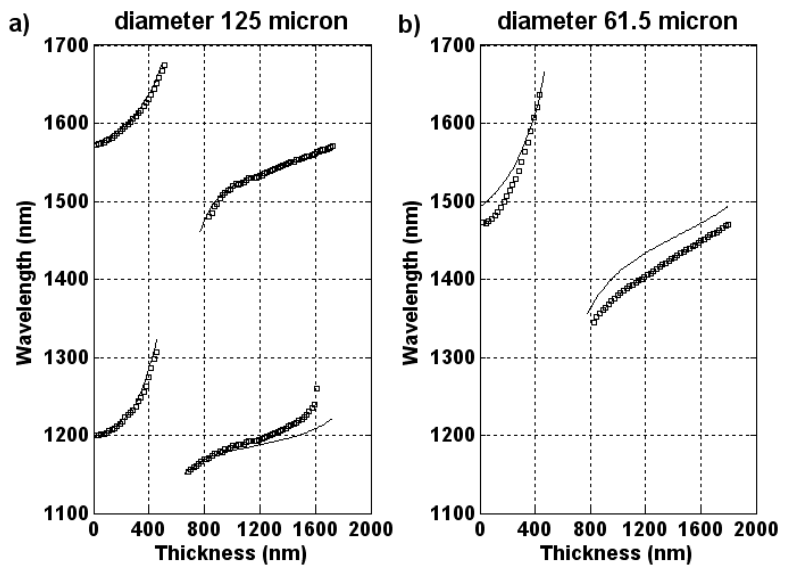

Fig. 12. Evolution as a function of thickness of the central wavelength of transmission bands obtained with an SMS structure where a PAH/PAA nanocoating is deposited. Simulation data, continuous line; experimental data, squares. a) MMF segment diameter $125 \mu \mathrm{m}$ and length $58 \mathrm{~mm}$ b) MMF segment diameter $61.5 \mu \mathrm{m}$ and length $15 \mathrm{~mm}$.

Once it has been proved that the sensitivity to nanocoating thickness is independent of MMF segment length, and that it increases if the diameter is reduced and the nanocoating refractive index is higher, some simulations will be performed, which will permit to obtain design rules for development of optimized devices for detecting variations of the surrounding medium refractive index and the nanocoating refractive index. The former is interesting for developing refractive index sensors, whereas the latter finds application in important fields such as chemical sensors [30].

Considering the basis of Fig. 9 and Fig. 12 three different structures will be compared: an SMS with an MMF segment of diameter $125 \mu \mathrm{m}$ and length $20 \mathrm{~mm}$ nanocoated with $\mathrm{TiO}_{2} / \mathrm{PSS}$, an SMS with an MMF segment of diameter $125 \mu \mathrm{m}$ and length $20 \mathrm{~mm}$ nanocoated with PAH/PAA, and an SMS with an MMF segment of diameter $61.5 \mu \mathrm{m}$ and length $20 \mathrm{~mm}$ nanocoated with PAH/PAA.

For the sake of simplicity a specific thickness of the nanocoating is analyzed. In view that the evolution of the transmission bands is different depending on the material, an $80 \%$ of the thickness where the fading region starts is selected. For the two structures with the PAH/PAA nanocoating this thickness is $400 \mathrm{~nm}$, whereas for the structure TiO2/PSS the thickness is $104 \mathrm{~nm}$.

In Fig. 13 by comparing the results obtained with the two structures of diameter $125 \mu \mathrm{m}$ it can be observed that the sensitivity to variations in the nanocoating refractive index is increased if a thin-film refractive index approaching that of the substrate is selected: PAH/PAA. This anomalous behavior, if compared with Fig. 9, can be understood by considering that transition region (the thickness range where a mode starts to be guided in the nanocoating) experiments a higher modification as the nanocoating refractive index approaches the refractive index of the MMF section (the substrate), which has been already proved for LPFGs [31].

In view that the best choice is the nanocoating of PAH/PAA, it is also presented in Fig. 13 the results obtained for an SMS with an MMF segment of diameter $61.5 \mu \mathrm{m}$ and length 20. In this case a $100.63 \mathrm{~nm}$ wavelength shift is obtained from -0.02 to 0.016 coating refractive index variation, whereas for the same structure but with a diameter of $125 \mu \mathrm{m}$ a wavelength shift of $45.34 \mathrm{~nm}$ is obtained. The ratio between theses values, like in the analysis of the sensitivity to nanocoating thickness, approximates the ratio between the diameters of the optical fiber.

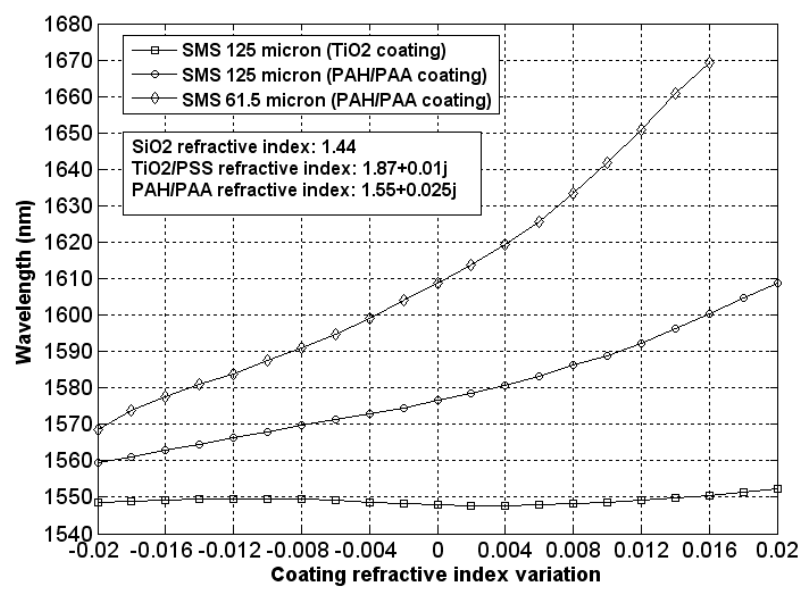

Fig. 13. Central wavelength of transmission bands obtained with an SMS structure as a function of nanocoating refractive index variation. Three different SMS structures are analyzed: MMF segment diameter $125 \mu \mathrm{m}$ and length $20 \mathrm{~mm}$ ( $\mathrm{TiO}_{2}$ coating), MMF segment diameter $125 \mu \mathrm{m}$ and length $20 \mathrm{~mm}$ (PAH/PAA coating) and MMF segment diameter $61.5 \mu \mathrm{m}$ and length $15 \mathrm{~mm}$ (PAH/PAA coating).

The analysis of the sensitivity to the surrounding medium is done in Fig. 14, 15 and 16. The same three SMS structures explored in Fig. 13 are analyzed and a new one is added (an SMS with an MMF segment of diameter $61.5 \mu \mathrm{m}$ and length $20 \mathrm{~mm}$ nanocoated with $\mathrm{TiO}_{2} / \mathrm{PSS}$ ), which permits to obtain a better knowledge of the behavior of refractometers based on thin-film coated SMS structures.

In Fig. 14, in order to obtain an analysis of a wide range of refractive indices, a $20 \%$ of the thickness where the fading region starts is selected. For the two structures with the PAH/PAA nanocoating this thickness is $100 \mathrm{~nm}$, whereas for the structure coated with $\mathrm{TiO}_{2} / \mathrm{PSS}$ the thickness is $26 \mathrm{~nm}$.

Two conclusions can be extracted. The first one is that the sensitivity increases as the surrounding medium refractive index approaches that of the substrate. The second one is that the material of the nanocoating is not important in terms of sensitivity, whereas the opposite is true for the selection of the diameter of the MMF section. The sensitivity obtained with a diameter of $61.5 \mu \mathrm{m}$ approximately doubles the sensitivity obtained with a diameter of $125 \mu \mathrm{m}$.

The simulations obtained in Fig. 15 and 16 for coating thickness values approaching the fading region permit to confirm the same conclusions obtained for Fig. 14. In addition to this, they permit observe that a better sensitivity is obtained as the thickness of the nanocoating increases, but at a cost of a smaller range of refractive indices that can be analyzed before the transmission band fades.

In order to compare the sensitivity of the refractometers, it will be analyzed the wavelength shift obtained for the same refractive index variation (1.333-1.36). In other words, the sensitivity will be obtained as the ratio between the wavelength shift and a refractive index range of 0.027 refractive index units. 
In Fig. 14, with coating thickness for PAH/PAA and $\mathrm{TiO}_{2} / \mathrm{PSS} 100 \mathrm{~nm}$ and $26 \mathrm{~nm}$ respectively (i.e. $20 \%$ of the value where the fading region starts), wavelength shifts of 4.7 , $11,4.5$ and $8.9 \mathrm{~nm}$ (i.e. sensitivities 174.1, 407.4, 166.7 and $329.6 \mathrm{~nm} / \mathrm{RIU}$ ) are obtained respectively for $\mathrm{TiO}_{2} / \mathrm{PSS}$ with a fiber diameter $125 \mu \mathrm{m}$, for $\mathrm{TiO}_{2} / \mathrm{PSS}$ with a fiber diameter $61.5 \mu \mathrm{m}$, for PAH/PAA with a fiber diameter $125 \mu \mathrm{m}$, and for PAH/PAA with a fiber diameter $61.5 \mu \mathrm{m}$.

In Fig. 15, with coating thickness for PAH/PAA and $\mathrm{TiO}_{2} / \mathrm{PSS} 250 \mathrm{~nm}$ and $65 \mathrm{~nm}$ respectively (i.e. $50 \%$ of the value where the fading region starts), wavelength shifts of 5.6, 15.8, 7.9 and $15.9 \mathrm{~nm}$ (i.e. sensitivities 207.4, 585.2, 292.6 and $588.9 \mathrm{~nm} / \mathrm{RIU}$ ) are obtained respectively for $\mathrm{TiO}_{2} / \mathrm{PSS}$ with a fiber diameter $125 \mu \mathrm{m}$, for $\mathrm{TiO}_{2} / \mathrm{PSS}$ with a fiber diameter $61.5 \mu \mathrm{m}$, for PAH/PAA with a fiber diameter $125 \mu \mathrm{m}$, and for PAH/PAA with a fiber diameter $61.5 \mu \mathrm{m}$.

In Fig. 16, with coating thickness for PAH/PAA and $\mathrm{TiO}_{2} / \mathrm{PSS} 400 \mathrm{~nm}$ and $126 \mathrm{~nm}$ respectively (i.e. $80 \%$ of the value where the fading region starts), wavelength shifts of $19.9,32.5,13.8$ and $34.5 \mathrm{~nm}$ (i.e. sensitivities 737, 1203.7, 511.1 and $1277.8 \mathrm{~nm} / \mathrm{RIU}$ ) are obtained respectively for $\mathrm{TiO}_{2} / \mathrm{PSS}$ with a fiber diameter $125 \mu \mathrm{m}$, for $\mathrm{TiO}_{2} / \mathrm{PSS}$ with a fiber diameter $61.5 \mu \mathrm{m}$, for PAH/PAA with a fiber diameter $125 \mu \mathrm{m}$, and for PAH/PAA with a fiber diameter $61.5 \mu \mathrm{m}$.

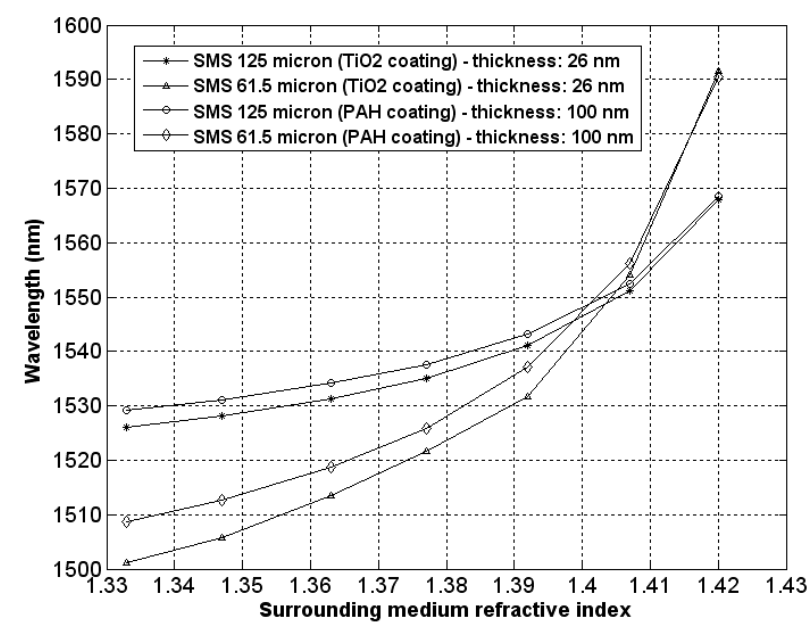

Fig. 14. Central wavelength of transmission bands obtained with an SMS structure as a function of surrounding medium refractive index. Coating thickness $20 \%$ of the value where the fading region starts. Four different SMS structures are analyzed: MMF segment diameter $125 \mu \mathrm{m}$ and length $20 \mathrm{~mm}\left(\mathrm{TiO}_{2}\right.$ coating), MMF segment diameter $61.5 \mu \mathrm{m}$ and length 15 mm $\left(\mathrm{TiO}_{2}\right.$ coating),MMF segment diameter $125 \mu \mathrm{m}$ and length $20 \mathrm{~mm}$ (PAH/PAA coating) and MMF segment diameter $61.5 \mu \mathrm{m}$ and length 15 mm (PAH/PAA coating).

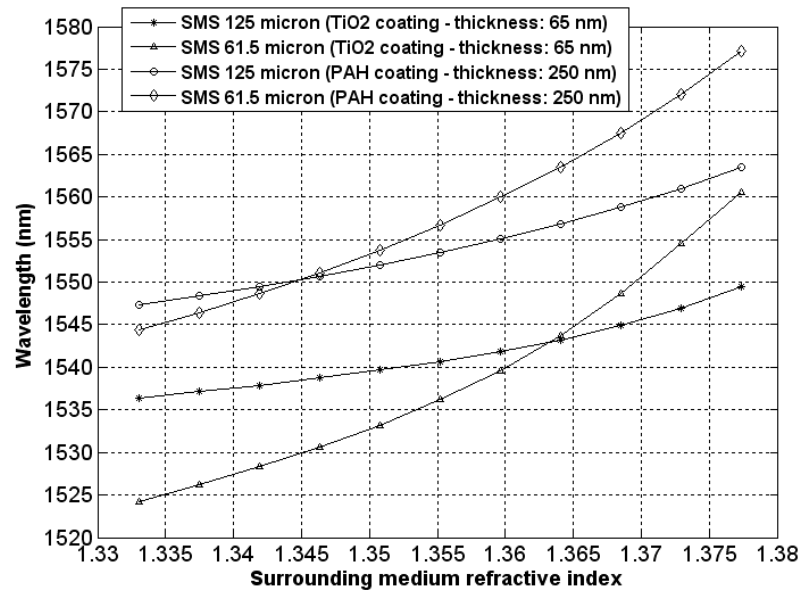

Fig. 15. Central wavelength of transmission bands obtained with an SMS structure as a function of surrounding medium refractive index. Coating thickness $50 \%$ of the value where the fading region starts. Three different SMS structures are analyzed: MMF segment diameter $125 \mu \mathrm{m}$ and length $20 \mathrm{~mm}\left(\mathrm{TiO}_{2}\right.$ coating), MMF segment diameter $61.5 \mu \mathrm{m}$ and length 15 mm ( $\mathrm{TiO}_{2}$ coating), MMF segment diameter $125 \mu \mathrm{m}$ and length $20 \mathrm{~mm}$ (PAH/PAA coating) and MMF segment diameter $61.5 \mu \mathrm{m}$ and length 15 mm (PAH/PAA coating).

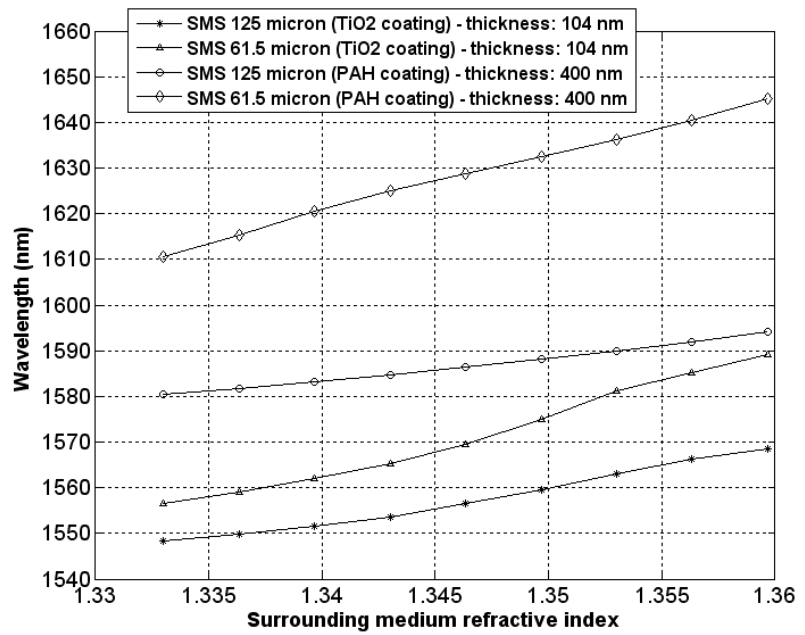

Fig. 16. Central wavelength of transmission bands obtained with an SMS structure as a function of surrounding medium refractive index. Coating thickness $80 \%$ of the value where the fading region starts. Three different SMS structures are analyzed: MMF segment diameter $125 \mu \mathrm{m}$ and length $20 \mathrm{~mm}\left(\mathrm{TiO}_{2}\right.$ coating), MMF segment diameter $61.5 \mu \mathrm{m}$ and length 15 mm $\left(\mathrm{TiO}_{2}\right.$ coating), MMF segment diameter $125 \mu \mathrm{m}$ and length $20 \mathrm{~mm}$ (PAH/PAA coating) and MMF segment diameter $61.5 \mu \mathrm{m}$ and length 15 mm (PAH/PAA coating).

\section{CONCLUSIONS}

In this work it has been analyzed both theoretically and experimentally the optimization of a single-mode-multimodesingle-mode (SMS) structure with a complex refractive index thin-film deposited on the cladding.

It has been proved that the length of the multimode-segment plays no role in the sensitivity of the bands obtained in the optical spectrum. However, the diameter of the multimodesegment is a very important parameter, which permits to increase the sensitivity of the device to variations in the thinfilm refractive index, thin-film thickness and surrounding 
medium refractive index.

The thin-film thickness is another important parameter which permits to set the attenuation bands in the optical spectrum in three different regions. In the middle region the attenuation bands fade, whereas for the other two regions, setting the coating thickness in the proximity of the fading region leads to a higher sensitivity to the thin-film refractive index, thin-film thickness and surrounding medium refractive index.

The thin-film refractive index plays also a role in the sensitivity of SMS coated devices. The sensitivity to thin-film thickness variations increases if the refractive index is higher, whereas the opposite is true for the sensitivity to variations in the refractive index of the thin-film. On the other hand, no major impact has been observed in the sensitivity to the surrounding medium refractive index for different thin-film refractive indices.

Finally, in view of the results obtained it is obvious that the sensitivity is increased if the surrounding medium refractive index is close to that of the substrate where the thin-film is deposited and if the thin-film thickness increases. This is something that has already been observed in other structures such as long-period fiber gratings.

By considering the previous five parameters and by using an adequate nanodeposition technique it should be possible, according to the final application the nanocoated SMS is used for, to develop optimized devices in research areas such as gas sensors, chemical sensors and biosensors, where uncoated SMS structures cannot be used.

\section{ACKNOWLEDGMENT}

This work was supported by the Spanish Ministry of Education and Science-FEDER TEC2010-17805 and Government of Navarra research grants.

\section{REFERENCES}

[1] S. Silva, E. G. P. Pachon, M. A. R. Franco, J. G. Hayashi, F. X. Malcata, O. Frazão, P. Jorge, C. M. B. Cordeiro, "Ultrahigh-sensitivity temperature fiber sensor based on multimode interference", App. Opt., vol. 51, pp. 3236-3242, 2012.

[2] L. B. Soldano, E. C. M. Pennings, "Optical multi-mode interference devices based on self-imaging: principles and applications," $J$. Lightwave Technol., vol. 13, pp. 615-627, 1995.

[3] L. L. Xue, L. Yang, "Sensitivity enhancement of RI sensor based on SMS fiber structure with high refractive index overlay", J. Lightwave. Technol., vol. 30, pp. 1463-1469, 2012.

[4] W. S. Mohammed, P. W. E. Smith, "All-fiber multimode interference bandpass filter," Opt. Lett., vol. 31, pp. 2547-2549, 2006.

[5] J. E. Antonio-Lopez, A. Castillo-Guzman, D. A. May-Arrioja, R Selvas-Aguilar, P. LiKamWa, "Tunable multimode-interference bandpass fiber filter," Opt. Lett., vol. 35, pp. 324-326, 2010.

[6] A. Mehta, W. Mohammed, E. G. Johnson, "Multimode interferencebased fiber-optic displacement sensor," IEEE Photon. Technol. Lett., vol. 15, pp. 1129-1131, 2003.

[7] Q. Wu, Y. Semenova, P. Wang, A. M. Hatta, G. Farrell, "Experimental demonstration of a simple displacement sensor based on a bent singlemode-multimode-single-mode fiber structure," Meas. Sci and Technol., vol. 22, 025203 (5pp), 2011.

[8] Q. Wu, Y. Semenova, A. M. Hatta, P. Wang, G. Farrell, "Single-modemultimode-singlemode fiber structures for simultaneous measurement of strain and temperature", Microw. And Opt. Tech. Lett., vol. 53, pp. 2181-2185, 2011
[9] V. I. Ruiz-Pérez, M. A. Basurto-Pensado, P. LiKamWa, J. J. SánchezMondragón, D. A. May-Arrioja, "Fiber Optic Pressure Sensor using Multimode Interference”, J. Phys.: Conf. Ser., vol. 274, 012025, 2011.

[10] Q. Wu, Y. Semenova, P. Wang, G. Farrell, "High sensitivity SMS fiber structure based refractometer - analysis and experiment" Opt. Express, vol. 19, pp. 7937-7944, 2011.

[11] I. Del Villar, C. R. Zamarreño, M. Hernáez, F. J. Arregui and I. R. Matías, "Lossy mode resonance generation with Indium Tin Oxide Coated Optical Fibers for sensing applications", J. Lightwave Technol. , vol. 28, pp. 111-117, 2010.

[12] N. D. Rees, S. W. James, R.P. Tatam, G. J. Ashwell, "Optical fiber longperiod gratings with Langmuir-Blodgett thin-film overlays", Opt. Lett., vol. 27, pp. 686-688, 2002.

[13] A.B. Socorro, I. Del Villar, J.M. Corres, I.R. Matias, F.J. Arregui "Lossy mode resonances dependence on the geometry of a tapered monomode optical fiber," Sens. Actuators A, vol. 180, pp. 25-31, 2012.

[14] J. Bravo, I. R. Matías, I. Del Villar, J. M. Corres, and F. J. Arregui, "Nanofilms on Hollow Core Fiber-Based Structures: An Optical Study," J. Lightwave Technol., vol. 24, pp. 2100-2107, 2006.

[15] L. Rindorf, J. B. Jensen, M. Dufva, L. H. Pedersen, P. E. Høiby, O. Bang "Photonic crystal fiber long-period gratings for biochemical sensing," Optics Express, vol. 14, pp. 8224-8231, 2006.

[16] T. Wang, S. Korposh, S. James, R. Tatam, S.-W. Lee, "Optical fibre long period grating sensor with a polyelectrolyte alternate thin film for gas sensing of amine odors," Sens. Actuators B, vol. 185, pp. 117-124, 2013

[17] C. R. Zamarreño, M. Hernáez, I. Del Villar, I. R. Matias and F. J. Arregui, "Tunable humidity sensor based on ITO-coated optical fiber," Sens. Actuators B, vol. 146, pp. 414-417, 2010.

[18] D. W. Kim, Y. Zhang, K. L. Cooper, A. Wang, "Fibre-optic interferometric immuno-sensor using long period grating", Electron. Lett., vol. 42, pp. 324-325, 2006

[19] A. B. Socorro, J. M. Corres, I. Del Villar, F. J. Arregui and I. R. Matias, "Fiber-optic biosensor based on lossy mode resonances," Sens. Actuators B, vol. 164, pp. 263-269, 2012.

[20] A. B. Socorro, J. M. Corres, I. Del Villar, F. J. Arregui and I. R. Matias, "Mode transition in complex refractive index coated single-modemultimode-single-mode structure" Optics Express, vol. 21, pp. 12668$12682,2013$.

[21] J. E. Antonio-Lopez,1,2 J. J. Sanchez-Mondragon,1 P. LiKamWa,2 and D. A. May-Arrioja3 "Fiber-optic sensor for liquid level measurement," Opt. Lett., vol. 36, pp. 3425-3427, 2011.

[22] B. H. Lee and J. Nishii, "Self-interference of long-period fibre grating and its application as temperature sensor," Electron. Lett., vol. 34, pp. 2059-2060, 1998

[23] G. W. Chern, L. A. Wang, "Transfer matrix method based on perturbation expansion for periodic and quasi-periodic binary longperiod fiber gratings," J. Opt. Soc. Am. A, vol. 16, pp. 2675-2689, 1999.

[24] P. Yeh, A. Yariv, and E. Marom, "Theory of Bragg fiber," J. Opt. Soc. Am., vol. 68, pp. 1196-1201, 1978.

[25] T. Erdogan, "Cladding-mode resonances in short and long period fiber gratings filters," J. Opt. Soc. Am. A, vol. 14, pp. 1760-1773, 1997.

[26] G. Decher, "Fuzzy Nanoassemblies: Toward Layered Polymeric Multicomposites," Science, vol. 277, pp. 1232-1237, 1997.

[27] I. Del Villar, I. R. Matias, F. J. Arregui, R. O. Claus, "Fiber-Optic Hydrogen Peroxide Nanosensor" IEEE Sens. J, vol. 5, pp. 365-371, 2005.

[28] M. Hernaez, I. Del Villar, C. M. Zamarreño, F. J. Arregui, I. R. Matias, "Optical fiber refractometers based on lossy mode resonances supported by TiO2 coatings" Appl. Opt., vol. 49, pp. 3980-3985, 2010.

[29] M. Giordanoa, M. Russo, A. Cusano, G. Mensitieri, G. Guerra, "Syndiotactic polystyrene thin film as sensitive layer for an optoelectronic chemical sensing device" Sens. Actuators B, vol. 109, pp. 177-184 2005.

[30] I. Del Villar, I. R. Matias, F. J. Arregui, P. Lalanne, "Optimization of sensitivity in Long Period Fiber Gratings with overlay deposition" Opt. Express, vol. 13, pp. 56-69 2005.

[31] I. Del Villar, I. R. Matias and F. J. Arregui, "Long-period fiber gratings with overlay of variable refractive index," IEEE Photon. Technol. Lett., vol. 17, pp. 1893-1895, 2005. 